\title{
A PRODUCT INTEGRAL REPRESENTATION FOR AN EVOLUTION SYSTEM
}

\author{
J. V. HEROD
}

ABSTRACT. This paper provides a product integral representation for a nonlinear evolution system. The representation is valid for expansive systems and provides an analysis in the nonexpansive case which is different from ones previously discovered.

In [7], D. Rutledge obtains a product integral representation for a nonexpansive, nonlinear semigroup. In [6], Neuberger gets such a representation for expansive semigroups by first considering nonexpansive evolution systems. This paper obtains a product integral representation for an expansive evolution system $M$. In this development, it is not required that $\lim _{h \rightarrow 0} h^{-1}[M(h, 0)-1] P$ exist. As a corollary to Theorem 3 , a statement equivalent to the statement that $M$ is nonexpansive is found.

Suppose that $\{G,+,|\cdot|\}$ is a complete, normed, Abelian group and that $S$ is the set of real numbers. If $f$ is a function from $S$ to $G$ and $a>b$, then denote the range of the restriction of $f$ to $[b, a]$ by $f([b, a])$. Also, the statement that $\left\{s_{p}\right\}_{0}^{n}$ is a subdivision of $\{a, b\}$ means that $s$ is a decreasing sequence with $s(0)=a$ and $s(n)=b$. The statement that $t$ is a refinement of the subdivision $s$ means that $t$ is a subdivision of $\{a, b\}$ and that there is an increasing sequence $u$ so that $s(p)=t(u(p))$ for $1 \leqq p \leqq n$. Finally, if $\left\{f_{p}\right\}_{1}^{n}$ is a sequence of functions from $G$ to $G$ and $g$ is in $G$, then

$$
\left[\prod_{p=1}^{n} f_{p}\right](g)=f_{1}\left(f_{2}\left(\cdots f_{n}(g)\right)\right) .
$$

An evolution system on $G$ is a function $M$ with domain contained in $S \times S$ so that if $x \geqq y$ then $M(x, y)$ is a function from $G$ to $G$ having the following properties:

(1) if $x \geqq y \geqq z$ then $M(x, y) M(y, z)=M(x, z)$ and $M(x, x)=1$, the identity function on $G$, and

(2) if $t$ is a number and $P$ is in $G$ then the function $g$ given by $g(x)$ $=M(x, t) P$, for all $x \geqq t$, is continuous.

Presented to the Society, January 23, 1970 under the title $A$ product integral representation for an expansive evolution system; received by the editors April 21, 1970.

AMS 1969 subject classifications. Primary 4750; Secondary 3495.

Key words and phrases. Product integral, evolution system, semigroups of operators.

Copyright (c) 1971, American Mathematical Society 
In order to obtain a product integral representation for the evolution system $M$, two additional conditions are used:

(3) there is an increasing, continuous function $\beta$ and a subset $D$ of $G$ so that

(a) if $P$ is in $D$ and $x>y$ then $M(x, y) P$ is in $D$, and

(b) if $P$ is in $D, \epsilon>0, a>b$, and $Q$ is in $M([b, a], b) P$ then there is a positive number $\delta$ so that if $R$ is in $M([b, a], b) P,|Q-R|<\delta$, and $a$ $\geqq x \geqq y \geqq b$, then

$$
|[M(x, y)-1] R-[M(x, y)-1] Q| \leqq[\exp (\beta(x)-\beta(y))-1] \cdot \epsilon,
$$

and

(4) there is a nondecreasing, continuous function $\alpha$ so that if $x>y$ and $\exp (\alpha(x)-\alpha(y))<2$, then $2-M(x, y)$ has range all of $G$ and, if $P$ and $Q$ are in $G$, then

$$
\begin{aligned}
{[2-\exp (\alpha(x)-\alpha(y))] \cdot|P-Q| } \\
\leqq|[2-M(x, y)] P-[2-M(x, y)] Q| .
\end{aligned}
$$

REMARK. It follows from condition (4) that if $\exp (\alpha(x)-\alpha(y))<2$, then $[2-M(x, y)]^{-1}$ has domain all of $G$, and if $P$ and $Q$ are in $G$ then

$$
\begin{aligned}
\mid[2-M(x, y)]^{-1} P- & {[2-M(x, y)]^{-1} Q \mid } \\
& \leqq[2-\exp (\alpha(x)-\alpha(y))]^{-1}|P-Q| .
\end{aligned}
$$

In this paper, the following three theorems are proved.

Theorem 1. Suppose that $P$ is in $D, a>b$, and $M$ satisfies conditions (1)-(4). It follows that $M(a, b) P=\prod_{a}^{b}[2-M]^{-1} P$-in the sense that if $\epsilon>0$, then there is a subdivision $s$ of $\{a, b\}$ so that if $\left\{t_{p}\right\}_{0}^{n}$ is a refinement of $s$ then

$$
\left|M(a, b) P-\prod_{p=1}^{n}\left[2-M\left(t_{p-1}, t_{p}\right)\right]^{-1} P\right|<\epsilon .
$$

Theorem 2. Suppose that $M$ satisfies conditions (1)-(4), if $x>y$ then $M(x, y)$ is continuous from $G$ to $G, D$ is dense in $G, a>b$, and $P$ is in $G$, it follows that $M(a, b) P={ }_{a} \prod^{b}[2-M]^{-1} P$.

Theorem 3. Suppose that $G$ is a Banach space, $M$ satisfies conditions (1)-(3). If $x>y$ then $M(x, y)$ is continuous from $G$ to $G, D$ is dense in $G$, and $\rho$ is a continuous, real valued function which is of bounded variation on each interval. These are equivalent:

(a) If $x>y$ and $P$ and $Q$ are in $G$ then

$$
|M(x, y) P-M(x, y) Q| \leqq \exp (\rho(x)-\rho(y)) \cdot|P-Q| \text {. }
$$


(b) If $x>y$ and $\exp (\rho(x)-\rho(y))<2$, then $2-M(x, y)$ has range all of $G$ and, if $P$ and $Q$ are in $G$, then

$$
\begin{aligned}
& {[2-\exp (\rho(x)-\rho(y))] \cdot|P-Q| } \\
& \leqq|[2-M(x, y)] P-[2-M(x, y)] Q| .
\end{aligned}
$$

IndiCATION OF Proofs. The following inequality is important in what follows; it may be established after considering the polynomial $P(z)=1-2 z^{2}+z^{3}$. It is labeled Lemma 1 for later reference.

LeMma 1. If $x$ is a number and $1 \leqq x \leqq(1+\sqrt{ } 5) / 2$ then $[2-x]^{-1} \leqq x^{2}$.

In the definitions and lemmas which follow, suppose that $M$ satisfies conditions (1)-(4), $a>b$, and $\epsilon>0$.

Definition. Define functions $\delta$ and $B$ as follows: if $P$ is in $D$ and $a \geqq z \geqq b$ then $\delta(z, P)$ is the largest number $d$ not exceeding 1 so that if $Q$ is in $M([z, a], z) P,|Q-R|<d$, and $a \geqq x \geqq y \geqq z$ then

$$
|[M(x, y)-1] Q-[M(x, y)-1] P| \because \leqq[\exp (\beta(x)-\beta(y))-1] \cdot \epsilon .
$$

Also, $B(z, P)$ is the largest number $u$ not exceeding $a$ so that if $u>v>z$ then $|M(v, z) P-P|<\delta(z, P)$.

REMARK. Note that the existence of $\delta$ follows from condition (3) and of $B$ follows from condition (2).

Lemma 2. Suppose that $P$ is in D. If $a \geqq x \geqq b,\left\{t_{p}\right\}_{0}^{n}$ is a subdivision of $\{B(x, P), x\}$, and $j$ is an integer in $[1, n]$, then

$$
\begin{aligned}
&\left|\left[M\left(t_{j-1}, t_{j}\right)-1\right] M\left(t_{j}, t_{n}\right) P-\left[M\left(t_{j-1}, t_{j}\right)-1\right] P\right| \\
& \leqq {\left[\exp \left(\beta\left(t_{j-1}\right)-\beta\left(t_{j}\right)\right)-1\right] \cdot \epsilon . }
\end{aligned}
$$

IndicAtion of Proof. If $\left\{t_{p}\right\}_{0}^{n}$ is a subdivision of $\{B(x, P), x\}$ and $j$ is an integer in $[1, n]$ then $x \leqq t_{j}<B(x, P)$. Thus $\left|M\left(t_{j}, x\right) P-P\right|$ $<\delta(x, P)$. Now, $M\left(t_{j}, x\right) P$ is in $M([x, a], x) P$, so if $a \geqq u \geqq v \geqq x$ then

$$
\begin{aligned}
&\left|[M(u, v)-1] M\left(t_{j}, x\right) P-[M(u, v)-1] P\right| \\
& \leqq[\exp (\beta(u)-\beta(v))-1] \cdot \epsilon .
\end{aligned}
$$

LEMma 3. Suppose that $P$ is in $D,\left\{t_{p}\right\}_{0}^{\infty}$ is an increasing sequence with values in $[b, a]$ and limit $z$. There is a positive integer $N$ so that if $n>N$ then $B\left(t_{n}, M\left(t_{n}, b\right) P\right) \geqq z$.

Indication of Proof. Suppose that $P$ is in $D$ and $t$ is an infinite increasing sequence with values in $[b, a]$ and limit $z$. The fact that $\left\{M\left(t_{p}, b\right) P\right\}_{p=0}^{\infty}$ converges in $G$ and has limit $M(z, b) P$ follows from 
condition (2). Let $Q$ be $M(z, b) P$. Since $Q$ is in $M([b, a], b) P$, there is a number $d$ so that $0<d<1$ and, if $|R-Q|<d$ and $R$ is in $M([b, a], b) P$ and $a \geqq x \geqq y \geqq b$, then

$$
|[M(x, y)-1] Q-[M(x, y)-1] R| \leqq[\exp (\beta(x)-\beta(y))-1] \cdot \epsilon / 2 .
$$

Let $w$ be so that if $z \geqq u \geqq w$ then $|Q-M(u, b) P|<d / 4$. Let $n$ be so that $t_{n}>w$. First, $\delta\left(t_{n}, M\left(t_{n}, b\right) P\right) \geqq d / 2$ because: suppose $R$ is in $M\left(\left[t_{n}, a\right], b\right) P$ and $\left|R-M\left(t_{n}, b\right) P\right|<d / 2$. Then $|R-Q|<d$ so that if $a \geqq x \geqq y \geqq b$ then

$$
\begin{aligned}
&\left|[M(x, y)-1] M\left(t_{n}, b\right) P-[M(x, y)-1] R\right| \\
& \leqq[\exp (\beta(x)-\beta(y))-1] \cdot[\epsilon / 2+\epsilon / 2] .
\end{aligned}
$$

Finally, $B\left(t_{n}, M\left(t_{n}, b\right) P\right) \geqq z$ because: suppose that $t_{n} \leqq v \leqq z$. Then

$$
\begin{aligned}
\left|M\left(v, t_{n}\right) M\left(t_{n}, b\right) P-M\left(t_{n}, b\right) P\right| \leqq\left|M\left(v, t_{n}\right) M\left(t_{n}, b\right) P-Q\right| \\
+\left|Q-M\left(t_{n}, b\right) P\right| \leqq d / 4+d / 4 \leqq \delta\left(t_{n}, M\left(t_{n}, b\right) P\right) .
\end{aligned}
$$

Lemma 4. Suppose that $P$ is in $D$. There is a subdivision $u$ of $\{a, b\}$ so that if $\left\{t_{p}\right\}_{0}^{n}$ is a refinement of $u$ and $p$ is an integer in $[1, n]$ then

$$
\begin{gathered}
\left|\left[M\left(t_{p-1}, t_{p}\right)-1\right] M\left(t_{p-1}, b\right) P-\left[M\left(t_{p-1}, t_{p}\right)-1\right] M\left(t_{p}, b\right) P\right| \\
\leqq\left[\exp \left(\beta\left(t_{p-1}\right)-\beta\left(t_{p}\right)\right)-1\right] \cdot 2 \epsilon .
\end{gathered}
$$

Indication of Proof. Suppose that $P$ is in $D$. By the previous lemma, there is a subdivision $\left\{u_{q}\right\}_{0}^{m}$ of $\{a, b\}$ so that if $q$ is an integer in $[1, m]$ then $u_{q-1}=B\left(u_{q}, M\left(u_{q}, b\right) P\right)$. Let $\left\{t_{p}\right\}_{0}^{n}$ be a refinement of $u$ and $p$ be an integer in $[1, n]$. Let $q$ be an integer in $[1, m]$ so that $u_{q-1} \geqq t_{p-1}>t_{p} \geqq u_{q}$. Then $\left|M\left(t_{p-1}, b\right) P-M\left(u_{q}, b\right) P\right|<\delta\left(u_{q}, M\left(u_{q}, b\right) P\right)$ and $\left|M\left(t_{p}, b\right) P-M\left(u_{q}, b\right) P\right|<\delta\left(u_{q}, M\left(u_{q}, b\right) P\right)$. Hence, if $a \geqq x \geqq y$ $\geqq u_{q}$, then

$$
\begin{gathered}
\left|[M(x, y)-1] M\left(t_{p-1}, b\right) P-[M(x, y)-1] M\left(t_{p}, b\right) P\right| \\
\leqq[\exp (\beta(x)-\beta(y))-1] \cdot 2 \epsilon .
\end{gathered}
$$

Indication of Proof of Theorem 1. Suppose that $P$ is in $D$. Let $u$ be a subdivision of $\{a, b\}$ as indicated in Lemma $4,\left\{s_{p}\right\}_{0}^{m}$ be a refinement of $u$ so that if $p$ is an integer in $[1, m]$ then $\exp \left(\alpha\left(s_{p-1}\right)-\alpha\left(s_{p}\right)\right)$ $<(1+\sqrt{ } 5) / 2$, and $\left\{t_{p}\right\}_{0}^{n}$ be a refinement of $s$. By Lemma 1 , if $p$ is an integer in $[1, n]$ and $P$ and $Q$ are in $G$, then

$$
\begin{aligned}
\mid\left[2-M\left(t_{p-1}, t_{p}\right)\right]^{-1} P- & {\left[2-M\left(t_{p-1}, t_{p}\right)\right]^{-1} Q \mid } \\
& \leqq \exp \left(2\left[\alpha\left(t_{p-1}\right)-\alpha\left(t_{p}\right)\right]\right) \cdot|P-Q| .
\end{aligned}
$$




$$
\begin{aligned}
& \mid \prod_{p=1}^{n}[\left.2-M\left(t_{p-1}, t_{p}\right)\right]^{-1} P-M(a, b) P \mid \\
&= \mid \sum_{j=1}^{n}\left\{\prod_{p=1}^{n+1-j}\left[2-M\left(t_{p-1}, t_{p}\right)\right]^{-1} M\left(t_{n+1-j}, b\right) P\right. \\
&\left.\quad-\prod_{p=1}^{n-j}\left[2-M\left(t_{p-1}, t_{p}\right)\right]^{-1} M\left(t_{n-j}, b\right) P\right\} \mid \\
& \leqq \\
& \quad \sum_{j=1}^{n} \exp \left(2\left[\alpha(a)-\alpha\left(t_{n+1-j}\right)\right]\right) \\
& \quad \cdot\left|M\left(t_{n+1-j}, b\right) P-\left[2-M\left(t_{n-j}, t_{n+1-j}\right)\right] M\left(t_{n-j}, b\right) P\right| \\
&=\sum_{j=1}^{n} \exp \left(2\left[\alpha(a)-\alpha\left(t_{n+1-j}\right)\right]\right) \\
& \quad \cdot \mid\left[M\left(t_{n-j}, t_{n+1-j}\right)-1\right] M\left(t_{n-j}, b\right) P \\
& \leqq \\
& \leqq \sum_{j=1}^{n}\left[\exp \left(2\left[\alpha(a)-\alpha\left(t_{n+1-j}\right)\right]\right) \cdot\left[\exp \left(\beta\left(t_{n-j}\right)-\beta\left(t_{n+1-j}\right)\right)-1\right] \cdot 2 \epsilon\right. \\
& \leqq \exp (2[\alpha(a)-\alpha(b)]) \cdot[\exp (\beta(a)-\beta(b))-1] \cdot 2 \epsilon .
\end{aligned}
$$

To see this last inequality, one should note Lemma 2.2 of [4].

Indication of Proof of Theorem 2. Suppose that $P$ and $Q$ are in $G, a>b$, and $\left\{t_{p}\right\}_{0}^{n}$ is a subdivision of $\{a, b\}$ so that, if $p$ is an integer in $[1, n]$, then $\left[2-M\left(t_{p-1}, t_{p}\right)\right]^{-1}$ has domain all of $G$.

$$
\begin{aligned}
\mid M(a, b) P-\prod_{p=1}^{n} & {\left[2-M\left(t_{p-1}, t_{p}\right)\right]^{-1} P|\leqq| M(a, b) P-M(a, b) Q \mid } \\
& +\left|\prod_{p=1}^{n}\left[2-M\left(t_{p-1}, t_{p}\right)\right]^{-1} Q-\prod_{p=1}^{n}\left[2-M\left(t_{p-1}, t_{p}\right)\right]^{-1} P\right| \\
& +\left|\prod_{p=1}^{n}\left[2-M\left(t_{p-1}, t_{p}\right)\right]^{-1} Q-M(a, b) Q\right| .
\end{aligned}
$$

Thus, if $D$ is dense in $G$ and $M(a, b)$ is continuous from $G$ to $G$, it follows from Lemma 1 that $M(a, b) P={ }_{a} \prod^{b}[2-M]^{-1} P$.

LEMMA 5. If $\rho$ is a continuous function from $S$ to $S$ and is of bounded variation on each interval of $S, a>b$, and $\epsilon>0$, then there is a subdivision $s$ of $\{a, b\}$ so that if $\left\{t_{p}\right\}_{0}^{n}$ is a refinement of $s$ then 


$$
\left|\exp (\rho(a)-\rho(b))-\prod_{p=1}^{n}\left[2-\exp \left(\rho\left(t_{p-1}\right)-\rho\left(t_{p}\right)\right)\right]^{-1}\right|<\epsilon .
$$

Indication of Proof. Notice that if $\rho$ is continuous and of bounded variation on each interval of $S, a>b$, and $\left\{t_{p}\right\}_{0}^{n}$ is a subdivision of $\{a, b\}$ so that, if $p$ is an integer in $[1, n]$, then $\exp \left(\rho\left(t_{p-1}\right)-\rho\left(t_{p}\right)\right)<2$ then

$$
\prod_{p=1}^{n}\left[2-\exp \left(\rho\left(t_{p-1}\right)-\rho\left(t_{p}\right)\right)\right]^{-1} \leqq \prod_{p=1}^{n}\left[2-\exp \left(\int_{t_{p}}^{t_{p-1}}|d \rho|\right)\right]^{-1} .
$$

With techniques similar to those used in the proof of Theorem 1 , it can be shown that, if

$$
\exp \left(\int_{t_{n}}^{t_{p-1}}\left|d_{\rho}\right|\right)<\frac{1+\sqrt{ } 5}{2} \quad \text { for } p=1,2, \cdots, n,
$$

then

$$
\begin{aligned}
& \left|\prod_{p=1}^{n}\left[2-\exp \left(\rho\left(t_{p-1}\right)-\rho\left(t_{p}\right)\right)\right]^{-1}-\exp (\rho(a)-\rho(b))\right| \\
& \leqq \exp \left(3 \int_{a}^{b}|d \rho|\right) \cdot \sum_{j=1}^{n}\left|\left[\exp \left(\rho\left(t_{n-j}\right)-\rho\left(t_{n+1-j}\right)\right)-1\right]^{2}\right| .
\end{aligned}
$$

The conclusion of the lemma follows.

Indication of Proof of Theorem 3. Suppose that $G$ is a Banach space and that $\rho$ is a function from $S$ to $S$ which is continuous and of bounded variation on each interval of $S$. Suppose also that $x>y$ and that $M(x, y)$ is a function from $G$ to $G$ having the property that if $P$ and $Q$ are in $G$ then $|M(x, y) P-M(x, y) Q| \leqq \exp (\rho(x)-\rho(y))|P-Q|$ $<2|P-Q|$. As in Lemma 1 of [5], let $X$ be in $G$ and $K(Z)$ be $.5[X+M(x, y) Z]$ for each $Z$ in $G$. Then $K$ is a contraction mapping and there is only one member $Z$ of $G$ so that $2 Z-M(x, y) Z=X$. Furthermore, if $P$ and $Q$ are in $G$, then

$$
\begin{aligned}
|Q-P| \leqq & .5|[2-M(x, y)] Q-[2-M(x, y)] P| \\
& +.5 \exp (\rho(x)-\rho(y))|P-Q| .
\end{aligned}
$$

Consequently, in Theorem 3, statement (a) implies statement (b). Finally, with $G$ and $\rho$ as supposed above, if $M$ satisfies conditions (1)-(3), $D$ is dense in $G$, statement (b) of Theorem 3 holds, and $x>y$, then, by Theorem $2, M(x, y) P={ }_{x} \Pi^{y}[2-M]^{-1} P$ for each $P$ in $G$ and, by Lemma 5, 
$\left|{ }_{x} \Pi^{y}[2-M]^{-1} P-{ }_{x} \prod^{y}[2-M]^{-1} Q\right| \leqq \exp (\rho(x)-\rho(y))|P-Q|$.

This completes the proof of Theorem 3.

\section{Examples.}

Example 1. Let $G$ be a Banach space and $T$ be a one-parameter semigroup of nonlinear transformations on $G$. That is, $T$ is a function from $[0, \infty)$ to the set of continuous transformations from $G$ to $G$ which satisfies

(1) $T(x) T(y)=T(x+y)$ if $x, y \geqq 0$,

(2) if $P$ is in $G$ and $g_{p}(x)=T(x) P$ for all $x$ in $[0, \infty)$ then $g_{p}$ is continuous and $\lim _{x \rightarrow 0^{+}} g_{p}(x)=P$,

(3) $|T(x) P-T(x) Q| \leqq|P-Q|$ if $x \geqq 0$ and $P$ and $Q$ are in $G$, and

(4) there is a dense subset $D$ of $G$ such that if $P$ is in $D$ then $g_{p}^{\prime}$ is continuous with domain $[0, \infty)$. By Theorem 2 , if $P$ is in $D$ and $x>0$, then $T(x) P={ }_{x} \prod^{0}[2-T(-d I)]^{-1} P$. Compare [5] and Theorem 2 of [7].

ExAmple 2. Let $f$ be an increasing function from the real numbers onto the real numbers so that $f^{\prime}$ is continuous and nonincreasing. Suppose also that $g$ is increasing and continuous, and that, for $x>y$ and $P$ a real number,

$$
M(x, y) P=f\left(g(x)-g(y)+f^{-1}(P)\right) .
$$

$M$ satisfies (1)-(4) but $\lim _{h \rightarrow 0^{+}} h^{-1}[M(h, 0)-1] P$ may not exist. Compare Example 2 of [8], Example 3.4 of [1], and Theorem A of [6].

Example 3. In case $M$ satisfies conditions (1) and (2) and if $P$ and $Q$ are in $G$ and $x>y$, then $|[M(x, y)-1] P-[M(x, y)-1] Q|$ $\leqq[\exp (\beta(x)-\beta(y))-1]|P-Q|$, then, according to [2] and [3], each value of $M$ has range all of $G$ and is invertible. This paper provides an alternate method for obtaining $M(x, y)^{-1}$.

\section{REFERENCES}

1. J. R. Dorroh, Some classes of semi-groups of nonlinear transformations and their generators, J. Math. Soc. Japan 20 (1968), 437-455. MR 37 \#6796.

2. J. V. Herod, Multiplicative inverses of solutions for Volterra-Stieltjes integral equations. Proc. Amer. Math. Soc. 22 (1969), 650-656.

3. - Coalescence of solutions for nonlinear Stieltjes equations, J. Reine Angew Math. (to appear)

4. J. S. MacNerney, Integral equations and semigroups, Illinois J. Math. 7 (1963), 148-173. MR $26 \# 1726$.

5. J. W. Neuberger, An exponential formula for one-parameter semi-groups of nonlinear transformations, J. Math. Soc. Japan 18 (1966), 154-157. MR 34 \#622. 
6. - Product integral formulae for nonlinear expansive semigroups and nonexpansive evolution systems, J. Math. Mech. 19 (1969), 403-409.

7. Dorothy Rutledge, $A$ generator for a semigroup of nonlinear transformations, Proc. Amer. Math. Soc. 20 (1969), 491-498.

8. G. F. Webb, Nonlinear evolution equations and product integration in Banach spaces, Trans. Amer. Math. Soc. 148 (1970), 273-282.

Georgia Institue of Technology, Atlanta, Georgia 30332 